\title{
MAPPIT 2: Second Generation High-resolution Imaging at the AAT
}

\author{
J. G. Robertson \\ School of Physics, University of Sydney, NSW 2006, Australia \\ and \\ European Southern Observatory, Karl-Schwarzschild-Str. 2, \\ D-85748 Garching, Federal Republic of Germany \\ g.robertson@physics.usyd.edu.au \\ Received 1995 May 25, accepted 1997 March 8
}

\begin{abstract}
Interferometric methods have been used at a number of observatories to improve the spatial resolution of large optical telescopes, approaching and in some cases reaching the diffraction limit. The principal methods used have been speckle interferometry and non-redundant masking (NRM). The MAPPIT (Masked APerture Plane Interference Telescope) instrument has been used for NRM observations at the $3.9 \mathrm{~m}$ Anglo-Australian Telescope. This paper describes a proposed instrument, MAPPIT 2, which would use a Shack-Hartmann wavefront sensor in parallel with an interferometer performing NRM or one-dimensional speckle interferometry. The inclusion of the data from the wavefront sensor will enhance the sensitivity of the instrument, especially for the imaging of relatively complex objects (those giving more than a few resolution elements with non-zero intensities). Limiting the instantaneous spatial resolution to one dimension allows available CCD detectors to operate with $100 \%$ duty cycle. Observations at a number of position angles allow two-dimensional images to be obtained.
\end{abstract}

Keywords: instrumentation: interferometers - stars: imaging, angular diameter

\section{Introduction}

A number of different approaches have been used to overcome the blurring effects of the Earth's turbulent atmosphere, and so obtain high-resolution images from large ground-based telescopes. The most commonly used methods have been speckle interferometry (Labeyrie 1970) and non-redundant masking (NRM; e.g. Wilson et al. 1992). Bedding, Robertson \& Marson (1994) described the MAPPIT interferometer at the Anglo-Australian Telescope (AAT), which has been used for NRM observations. At present a number of adaptive optics (AO) systems are being developed and brought into use. AO differs from interferometric methods in that wavefront distortions imposed by the atmosphere are sensed and removed in real time, allowing a highresolution image to be formed on a long-exposure detector or to be placed on a spectrograph entrance aperture.

Results to date from speckle interferometry and NRM have concentrated on binary and multiple star separations and orbits, and measurements of stellar diameters and surface features for cool giants with disks resolvable by $4 \mathrm{~m}$ telescopes. The restriction of current optical interferometric methods to relatively simple objects, showing at most a small number of non-zero resolution elements along each axis, stems from the nature of the available data. Because turbulence in the Earth's atmosphere produces decorrelation of phases over spatial scales larger than about $10 \mathrm{~cm}$ (the Fried length, $r_{0}$ ), and over timescales longer than about $10 \mathrm{~ms}$, it is not possible to measure directly the interference fringe visibilities and phases for the various baselines, as is done in a phase-stable radio synthesis telescope. Instead, methods equivalent to autocorrelation (or calculation of the power spectrum) are used to extract fringe visibility amplitudes, resulting in a response proportional to $V^{2}$, where $V$ is the true object fringe visibility. For a well resolved (i.e. complex) object, many of the intermediate- and long-baseline fringes will have visibilities of order $1 \%$, and these become too weak to be measured satisfactorily in the presence of noise when the system response is proportional to $V^{2}$.

Phase information is also required to construct a true image, and is normally extracted in the form of the closure phase, which is the argument of the complex bispectrum (e.g. Haniff 1989). In the case of speckle interferometry the use of the bispectrum is termed speckle masking (Weigelt et al. 1986). The bispectrum depends on the triple correlation around a triangle of baselines; thus it is proportional to $V^{3}$ (or 
closer to $V^{2}$ if one short baseline is included). While the resulting closure phase has the desirable property of independence from atmospherically induced phase corruption, the dependence on $V^{3}$ results in the signal becoming difficult or impossible to detect in the presence of noise for low-visibility baselines. For example, the surface features on red giant stars were difficult observations for interferometry even though the stellar disks were only resolved into about $2 \times 2$ beamwidths (FWHM), and the target stars were bright.

Although they also work through the turbulent atmosphere, AO systems are not subject to the same limitations, because they incorporate a wavefront sensor. Within certain constraints, the wavefront data enable the imaging to be done in an effectively phase-stable manner. AO systems, however, are complex and expensive to set up. They generally aim for full diffraction-limited resolution only in the infrared, and/or on intermediate-sized telescopes, because working at longer wavelengths or with a smaller primary aperture reduces the number of sensors and actuators needed. It is possible to reach fainter objects in the infrared, thus extending the sky coverage (e.g. Beckers 1993).

\section{Post-detection Turbulence Compensation}

It is against the above background that the technique of 'post-detection turbulence compensation' has been developed (Primot, Rousset \& Fontanella 1990; Gonglewski \& Dayton 1992). This method uses a wavefront sensor to find the instantaneous phase corruption imposed by the atmosphere (on the usual timescales of about $10 \mathrm{~ms}$ ) but instead of using the corrections to drive a deformable mirror, they are used within the data reduction process to aid imaging from data that are acquired as speckle interferograms. This has the advantage of eliminating the costly and difficult construction of a deformable mirror system; there are a number of other advantages as well. On the debit side, the 'science detector' must (like the wavefront sensor) use numerous short exposures, and the data reduction system is more complex. Provided that there is enough signal for the wavefront sensor to operate, the science detector data can be treated in a manner analogous to those from a phase-stable interferometer, avoiding the $V^{2}$ and $V^{3}$ dependencies.

Several systems incorporating post-detection turbulence compensation have been used or are under development (see Andersen 1992; Marais et al. 1992). They have used full two-dimensional coverage of the telescope aperture. The fully filled aperture results in two-dimensional speckle interferograms on the science detector. While this is optimum in respect of the baseline coverage of the interferometer, it has a drawback due to limitations of the currently available detectors for two-dimensional images. These either have low quantum efficiency (image intensifiers) or a low duty cycle and significant read noise for such short images (CCDs with a shutter).

The instrument proposed here, called 'MAPPIT 2 ', uses the same principle, but in a one-dimensional array. By going to one dimension, the data take a form that can be read out by CCD detectors with a tolerable level of read noise and in a sufficiently short period that a $100 \%$ duty cycle is possible. The penalty is that observations must be taken at a number of different position angles on the sky, which increases the overall duration of the observation of one object. The different position angles are easily obtained using optical beam rotation. The novel features of MAPPIT 2 are the use of postdetection turbulence compensation in combination with aperture masking and one-dimensional operation which allows a $100 \%$ duty cycle.

It is proposed to implement the MAPPIT 2 system at the coudé focus of the AAT. It would be complementary to the AO system being constructed by a consortium of Australian institutions, in that the AO system will perform infrared imaging, whereas MAPPIT 2 will aim for the full diffraction-limited resolution in the visible range [a FWHM as small as $\sim 20$ mas (milliarcsec) for objects that can be observed successfully at $\lambda 400 \mathrm{~nm}$ and at the maximum baseline of $3.9 \mathrm{~m}$ ].

\section{Outline of Design}

Figure 1 shows schematically the proposed design of MAPPIT 2. The instrument may be divided into a number of subsystems.

\subsection{Beamsplitter}

The wavefront sensor must view the same slit of the aperture as will fall on the mask to form speckles or fringes on the science detector. Thus the wavefront sensor and the mask must both be in planes conjugate to the telescope pupil (or the dominant atmospheric turbulent layer), and must be optically superimposed. This may be achieved using a conventional beamsplitter, as shown. However, for greater efficiency it would be preferable for the wavefront sensor to receive all the light except the narrow band used by the science detector arm. With a suitably rearranged layout, this may be implemented as a later development, employing a narrowband mirror (rugate filter) or possibly using the reflection from the narrowband filter itself.

\subsection{The Wavefront Sensor}

It is proposed to use a Shack-Hartmann sensor, which will consist of a one-dimensional row of about 40 lenslets, each of order $0.5 \mathrm{~mm}$ in size. Each lenslet subtends a subaperture of $r_{0} \sim 10 \mathrm{~cm}$ ) or slightly less on the primary mirror. The image spot formed by a lenslet has a displacement from its nominal position 


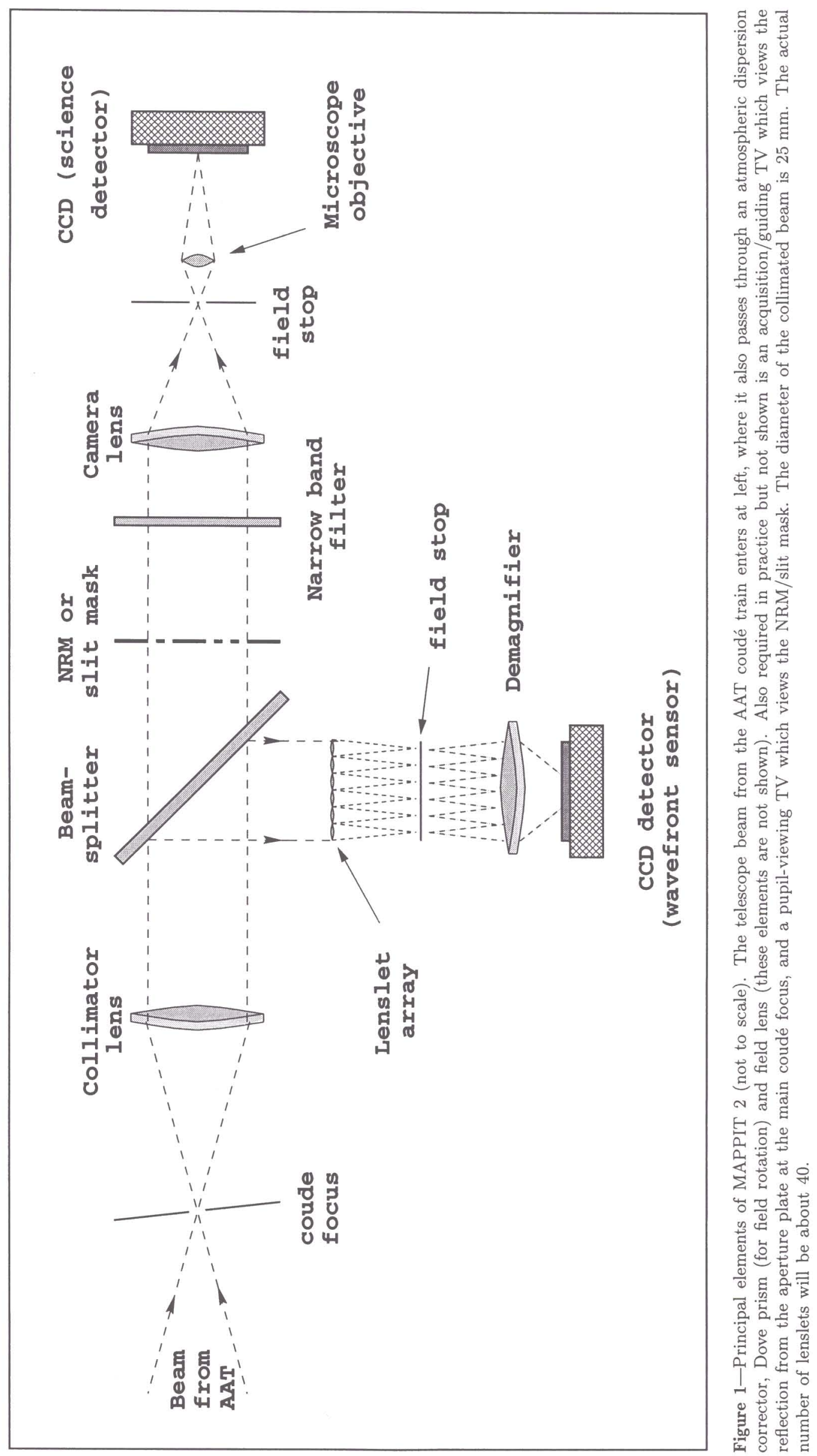


which reveals the average phase slope across the corresponding part of the aperture. From the set of phase slopes at roughly $r_{0}$ spacings, for each exposure of $\sim 10 \mathrm{~ms}$ the full wavefront phase profile can be found, and hence the instantaneous pointspread function (PSF) of the telescope/atmosphere combination (e.g. Marais et al. 1992). Real-time estimates of the atmospheric turbulence parameters $r_{0}$ and $t_{0}$ plus residual focus errors can also be found from the sensor data.

The Shack-Hartmann sensor can use broadband light, thus allowing the system to obtain adequate signal (rms phase errors $<0 \cdot 3 \mathrm{rad}$, corresponding to 100 detected photons per lenslet spot) for objects down to about $9.0 \mathrm{mag}$. This value assumes observation of a red star, with $10 \mathrm{~cm}$ apertures, $10 \mathrm{~ms}$ exposure time, a $50 \%$ beamsplitter, and the AAO Thomson CCD as detector. Since the system is one-dimensional, only the spot displacements along the line of the array are needed, so the illuminated area of the CCD can be binned along columns to give a one-dimensional output array. Originally introduced for NRM observations (Buscher et al. 1990), column binning results in CCD readout times of order $10 \mathrm{~ms}$, enabling the detectors to be run without a separate shutter and achieving $100 \%$ duty cycle. At the same time, the concentration of detected photons from a whole column into a single pixel reduces the effects of readout noise on the data.

For square lenslets (the preferred outline) forming a diffraction-limited spot on the CCD, the spot FWHM is $\lambda f / d$, where $\lambda$ is the wavelength and $f / d$ is the focal ratio of the individual lenslets. In order to obtain spots large enough for Nyquist sampling by $19 \mu \mathrm{m}$ CCD pixels it is necessary to use focal ratios of 100 or more. The alternative of precisely aligning a smaller lenslet spot on the pixel boundaries of a quadrant detector is not feasible for the number of lenslets required. The displacements that must be measured are small, since a phase slope of $2 \pi$ radians across one lenslet subaperture produces only a shift of the spot equal to its diffraction FWHM. The predominant effect of the atmospheric seeing is to move the spots around, not to broaden them. However, the spots will be broadened somewhat by chromatic aberration, residual wavefront curvature over the subapertures, changes in the atmospheric phases during the sampling time, and object structure if the target object has a size comparable with the seeing disk.

For maximum speed of the CCD readout from the wavefront sensor, and to fit within available CCD sizes, the spots should be placed as close to each other as possible, while still allowing adequate spacing to cope with maximum expected phase slopes. It can be shown that a value of $\pm 7.5 \mathrm{rad}$ phase difference across one subaperture allows for the maximum effects of seeing, with some reserve for the spot-broadening effects mentioned above. A key parameter of any lenslet array, independent of the optical system used to feed it, is the quantity $d^{2} / \lambda f$. It gives the ratio of spot separation to spot size (FWHM), and to accommodate the maximum phase difference of $\pm 7.5 \mathrm{rad}$ across a lenslet, this parameter should be 4.4 (at the longest usable wavelength). This is equivalent to 8.8 pixels per lenslet on the detector. Minor telescope tracking errors may cause larger displacements of the spots, but in this case all spots move in a correlated manner, so they can be followed as a group if sufficient extra pixels are allowed at the edges of the detector.

\subsection{The Interferometry Subsystem}

The wavefront sensor input comes from a slit across the telescope pupil. It will have a maximum length of $3.3 \mathrm{~m}$ for observations in which it is essential to avoid the central obstruction, or $3.9 \mathrm{~m}$ for those in which a central gap can be accepted. The beamsplitter passes light from the same slit to either an array of holes for formation of NRM fringes on the science detector, or to a slit for use of slit speckle interferometry. An interference filter selects the required narrow wavelength band. NRM will probably be preferred for objects bright enough to give adequate signal, because the baseline redundancy of speckle interferometry reduces the relative amplitude of the higher spatial frequencies in the data. The science detector will be another CCD, which should be operated with readout synchronised to the wavefront sensor readout. Column binning will again be needed for adequate readout speed, and is allowable because the fringes or speckles have only one-dimensional structure.

\section{Data Processing}

The availability of the instantaneous PSF will make it possible to treat the fringe or speckle data differently from the usual methods. It will be possible to form coherent sums of the (Fourier-transformed and phase-compensated) fringes or speckles from the science detector, without using the higher correlations (power spectra and bispectra) which result in higher-order dependence on the object visibilities and the attendant difficulties for well resolved objects. In summing data over say 10000 frames (each about $10 \mathrm{~ms}$ ) to reveal usable signals, it is assumed that the wavefront sensor receives an adequate signal in each frame, but its broadband operation and the limited number of pixels used to receive each spot give a suitably faint limiting magnitude.

The first stage of data processing is the formation of the PSF from each data frame of the wavefront sensor. Marais et al. (1992) discussed a Fourier method which takes into account the discrete sampling imposed by 
the wavefront sensor lenslet array. In the cases where the telescope's central obstruction causes a gap in the wavefront sensor data, iterative model-fitting can be used to make the necessary one-parameter interpolation of the phase across the gap, but only for objects with simple structures. Similar methods have been verified in the processing of data from the present MAPPIT instrument. For complex objects, continuous wavefront sensor coverage along the aperture slit will be needed.

The second stage is the coherent summation of all exposures at one position angle. Information from the PSF is used to phase-shift the fringes (or Fourier components of speckles) from the science detector back to standard registrations, so that the exposures can then be summed without having to detect the fringes or speckles in individual exposures. This process has been described by Primot et al. (1990) and Gonglewski \& Dayton (1992). Enhancements to be used in the MAPPIT 2 procedure will be described in a later publication.

The result will be a one-dimensional data set representing the true object as convolved with a smooth beam of width appropriate to the spatial resolution, with both additive and multiplicative noise. The noise in the estimate of the PSF from the wavefront sensor has the undesirable property that it depends on the object structure, but in practice it is not expected to be the dominant noise contribution.

The final stage of the process is to combine the separate one-dimensional observations into a single image, using a conventional Fourier transform, and CLEAN or MEM techniques to remove the artifacts due to limited position-angle sampling. The methods are well known, having been developed for processing data from linear radio synthesis arrays. In NRM mode, the data from MAPPIT 2 will form discrete points in the $u, v$ plane, analogous to the data from a phase stable radio array. They will be distributed along radial spokes, but can be treated as a whole when making the image because the phase of each complex visibility point has been referred to a fixed zero point.

For studies of barely resolved objects, it will be possible to examine individual fringe visibilities as a function of baseline by simply using the coherent sums of one-dimensional Fourier transformed data at each position angle.

\subsection{Calibration}

The calibration procedure for the system will be a twostage process. First, observations of an unresolved pinhole at the coudé focus, with illumination by a tungsten lamp, will give wavefront sensor spot positions marking the zero points from which spot deviations will be measured. Likewise the fringe phase on the science detector gives the zero point for each baseline on that detector. The pinhole position in the focal plane thus becomes the zero phase reference position. This calibration procedure will remove the effects of lenslet imperfections and static aberrations of the telescope and optical system. Second, observations of unresolved standard stars will be used to determine the effects of residual visibility loss due to the non-zero sampling time and the non-zero spacing of the wavefront sensor elements across the aperture.

\section{Sensitivity Limits}

The wavefront sensor has a limit near 9th magnitude, as noted above. In general it is expected that the overall sensitivity limit will be imposed by the readout noise from the science detector, even given that coherent 'blind' integration is possible. The principal reason for this is that the speckle or fringe data on the science detector must be narrow band in order to avoid smearing the fringes ${ }^{1}$, and a large number of pixels $(\sim 200)$ must be used in order to adequately sample all the fringe frequencies. The sensitivity limit depends on the complexity of the object structure, since a very high initial signal to noise ratio (SNR) of the combined fringe (or speckle) data is required to achieve even a modest final SNR for a baseline which has very low visibility due to resolved object structure. As an example, a uniform circular disk resolved into 10 pixels across the diameter has a theoretical fringe visibility that goes through 9 extrema beyond the central maximum, which have visibilities ranging from 10 to $1 \%$. To achieve a SNR of 3 for the baseline with $1 \%$ visibility requires an initial SNR of at least 300 . For an observing bandwidth of $20 \mathrm{~nm}$; a CCD readout noise of $5 \mathrm{e}^{-}$; NRM with 5 holes, each $10 \mathrm{~cm}$ square; and an observing time of $1000 \mathrm{~s}$ per position angle, the limit will be at 4.5-5.5 mag. Using slit speckle and/or a wider wavelength band or larger holes would gain in photon rate but lose further fringe visibility due to fringe smearing. Trials and simulations will be needed to find the optimum strategy. The above limit is almost entirely due to CCD readout noise, and if essentially noise-free detectors became available (but retaining the quantum efficiency and count-rate ability of CCDs) then the limit would improve by 3 magnitudes.

Thus we expect that a system using presently realisable detectors would be limited to about 4.5$5.5 \mathrm{mag}$ for the most complex feasible objects, viz. about 10 resolution elements along each axis. But for simpler, less well resolved objects, the limit would extend to 7-8 mag, depending on the actual object structure and the dynamic range required.

\footnotetext{
${ }^{1}$ Wavelength-dispersed systems would be possible with a fast two-dimensional detector; cf. Bedding et al. (1994).
} 


\section{Scientific Program}

The principal targets for which MAPPIT 2 will be suitable are similar to those that have been observed by existing speckle and NRM facilities, namely diameters and surface features of red giant and Mira stars, and study of binary and multiple star systems. But MAPPIT 2 is expected to be able to make successful observations of fainter objects, to have higher dynamic range, and to observe at wavelengths presently unusable due to sensitivity limitations (e.g observation of red giants in the blue, where diameter measurements are particularly useful because of reduced absorption blanketing, and the angular resolution from a given telescope is greatest). The ability to image more complex objects would be appropriate for well resolved stars and perhaps the brightest asteroids.

MAPPIT 2 would be ideal for the 'large-scale' checkout of fields observed by the SUSI long-baseline interferometer (Davis 1994). SUSI is insensitive to companions with separations greater than about 70 mas, whereas MAPPIT 2 would readily find companions at separations between 70 mas and the seeing disk size, down to a magnitude limit similar to that of SUSI.

MAPPIT 2 would also be suitable for imaging of bright novae (and supernovae), especially in the early phases with high brightness and small angular diameter.

\section{Conclusion}

The combination of a one-dimensional wavefront sensor with non-redundant masking or slit speckle interferometry, using the wavefront data to carry out post-detection turbulence compensation in the data processing phase, promises a significant advance in high-resolution imaging at reasonable cost. An instrument such as MAPPIT 2 would be able to image bright objects of moderate complexity (greater than for present passive interferometric methods, but much less than HST), at the highest angular resolution obtainable from single telescopes.

There are several developments of this project which could follow at a later stage. The method would be well suited for use with an 8 or $10 \mathrm{~m}$ telescope, yielding correspondingly improved angular resolution. A larger number of lenslets would be required in the wavefront sensor and larger number of pixels in both wavefront and science detectors, although for the wavefront sensor this increase would be lessened if the site had better seeing. Another future possibility would be use with laser guide stars, which could provide the signal needed for the wavefront sensor even for stars fainter than 9 mag. But such a development would be of limited use, certainly for the imaging of complex objects, unless detectors with much lower read noise also become available. If fast detectors with negligible read noise do become available, they would greatly extend the range of objects accessible to high-resolution imaging as described here.

\section{Acknowledgments}

Ana Torres-Dodgen kindly supplied calibrated spectrophotometry of $\mathrm{M}$ stars which assisted in the sensitivity calculations. Tim Bedding provided helpful comments.

Andersen, T. (ed.) 1992, Wavefront-supported Post-facto Image Correction, Nordic Optical Telescope Scientific Association Miniworkshop

Beckers, J. M. 1993, ARA\&A, 31, 13

Bedding, T. R., Robertson, J. G., \& Marson, R. G. 1994, A\&A, 290, 340

Buscher, D. F., Haniff, C. A., Baldwin, J. E., \& Warner, P. J. 1990, MNRAS, 245, 7P

Davis, J. 1994, Very High Angular Resolution Imaging, ed. J. G. Robertson and W. J. Tango (Dordrecht: Kluwer), p. 135

Gonglewski, J. D., \& Dayton, D. 1992, in Wavefrontsupported Post-facto Image Correction, ed. T. Andersen (Nordic Optical Telescope Scientific Association Miniworkshop), p. 18

Haniff, C. A. 1989, in Diffraction-limited Imaging with Very Large Telescopes, ed. D. M. Alloin \& J.-M. Mariotti (Dordrecht: Kluwer), p. 171

Labeyrie, A. 1970, A\&A, 6, 85

Marais, T., Michau, V., Fertin, G., Primot, J., \& Fontanella, J. C. 1992, in High Resolution Imaging by Interferometry II, ed. F. Merkle (Munich: European Southern Observatory), p. 589

Primot, J., Rousset, G., \& Fontanella, J. C. 1990, J. Opt. Soc. Am. A, 7, 1598

Weigelt, G., Baier, G., Ebersberger, J., Fleischmann, F., Hofmann, K.-H., \& Ladebeck, R. 1986, Opt. Eng., 25, 706

Wilson, R. W., Baldwin, J. E., Buscher, D. F., \& Warner, P. J. 1992, MNRAS, 257, 369 